\title{
Follow-up study of 6.5 years of admissions to a UK female medium secure forensic psychiatry unit
}

\author{
John Tully, ${ }^{1}$ Alessandra Cappai, ${ }^{2}$ John Lally, ${ }^{3}$ Maria Fotiadou ${ }^{2}$
}

BJPsych Bulletin (2019) 43, 54-57, doi:10.1192/bjb.2018.77

${ }^{1}$ Department of Forensic and Neurodevelopmental Sciences, Institute of Psychiatry, Psychology and Neuroscience, Kings College London; ${ }^{2}$ South London and Maudsley Foundation Trust Forensic Services; ${ }^{3}$ Department of Psychosis Studies, Institute of Psychiatry, Psychology and Neuroscience, Kings College London and Department of Psychiatry, Royal College of Surgeons in Ireland

Correspondence to John Tully (john. tully@kcl.ac.uk)

First received 5 Feb 2018, final revision 16 Aug 2018, accepted 21 Aug 2018

(c) The Authors 2018. This is an Open Access article, distributed under the terms of the Creative Commons Attribution-NonCommercialNoDerivatives licence (http:// creativecommons.org/licenses/by-ncnd/4.0/), which permits noncommercial re-use, distribution, and reproduction in any medium, provided the original work is unaltered and is properly cited. The written permission of Cambridge University Press must be obtained for commercial re-use or in order to create a derivative work.
Aims and method We aimed to examine clinical and risk outcomes at follow-up, and reoffending and readmission rates, for a sample of 50 admissions to a female medium secure unit (MSU). Demographic, clinical risk assessment (HCR-20 and HoNOS-Secure) and quality of life data were collected using validated measures for all admissions to a female MSU ward in London, UK, between April 2008 and November 2014.

Results All clinical and risk assessment scale scores had improved at follow-up. Quality of life compared favourably to community samples and was good for physical, social and environmental factors and acceptable for psychological health. Twenty-six per cent had at least one readmission, while $17.5 \%$ reoffended in the period studied. A longer duration of admission and use of restrictions on discharge was associated with reduced reoffending, but not readmission.

Clinical implications Admission is associated with improvement on clinical risk assessment at follow-up. Longer hospital admissions and use of restrictions on discharge may be necessary to prevent reoffending in this group.

\section{Declaration of interest None.}

Keywords Women offenders; quality of life; reoffending; readmission; medium security.
Forensic psychiatry services are low-volume, high-cost services which seek to balance clinical treatment for public protection with caring for vulnerable patient groups. ${ }^{1}$ In the UK, forensic psychiatric in-patient care is organised into three levels: high, medium and low secure facilities. ${ }^{2}$ Patients are admitted to a specified level of security based on forensic history, level of risk to self or others, and diagnosis of a mental disorder. On discharge from in-patient services, many patients are subject to conditions under a Community Treatment Order (CTO), or 'restrictions' under Section 41 of the Mental Health (MHA) 1983, as amended in 2007 (for England and Wales), known as 'conditional discharge'. After discharge, such patients may leave hospital, but if they breach conditions or restrictions, they may be recalled to hospital. Examples of conditions and restrictions include being resident at a specified address, medication adherence, or cooperating with supervision by mental health services. Prevention of reoffending after discharge and reduction in readmission rates are key roles of forensic services. It is therefore essential that services be aware of factors involved in reoffending and readmission.

In the past decade, there has been a six-fold increase in the number of women admitted to medium secure forensic psychiatry services $\left(\mathrm{MSUs}^{3}\right)$. Approximately $12-15 \%$ of medium secure beds in the UK are occupied by women. ${ }^{2,4,5}$ Despite this, there remains a paucity of demographic and clinical data on female MSU patients, particularly in relation to progress after discharge. Using a longitudinal cohort design, we assessed clinical risk assessment follow-up data and quality of life, using validated measures, in a female MSU sample. We also sought to identify clinical and demographic factors associated with reoffending and readmission rates following hospital discharge.

\section{Methods}

\section{Sample and study setting}

This study comprised all patients admitted to Spring Ward, the female ward of River House MSU in South London and Maudsley Foundation (SLaM) Trust, between April 2008 and November 2014 (6.58 years). Spring Ward contains 16\% of the beds within the unit. The service covers a population of 1090544 people in London, approximately $50 \%$ of whom are female. Spring Ward opened in April 2008 and employs a multidisciplinary biopsychosocial approach to support women in 
their pathway of recovery and reintegration into the community. Ethical approval for this project was sought and obtained from the SLaM Trust Research and Ethics Committee.

\section{Data collection}

We examined the electronic clinical records of all patients. Demographic, clinical and risk assessment data are collected routinely at admission and throughout the course of admission. Standard risk assessment during MSU admission in the UK includes the Historical Clinical Risk Management-20 $\left(\mathrm{HCR}-20^{6}\right)$, Health of the Nation Outcome Scale (HoNOS), and Health of the Nation Outcome Scales for Users of Secure and Forensic Services (HoNOS-Secure ${ }^{7}$ ). The predictive validity for violence of the HCR-20 has been established in female patients in medium secure settings, ${ }^{8}$ and HoNOS has previously been used to identify clinical improvements following admission to female MSUs. ${ }^{9}$ All patients were assessed using these scales at the time of their admission, or shortly after. All patients were reassessed using the HCR-20, HoNOS and HoNOS-Secure scales between April and October 2015. Readmission and reoffending rates following initial discharge were collated, based on SLaM data. Quality of life was measured using the World Health Organization Quality of Life Scale, brief version (WHOQOL-BREF ${ }^{10}$ ), which has been validated in large international samples, including those with mental illness. ${ }^{11}$ One assessment was obtained for each patient, close to or soon after discharge.

\section{Statistical analysis}

Comparisons between the groups were conducted using $\chi^{2}$-tests for categorical variables and $t$-tests for continuous variables. All statistical tests were two-sided, and the $\alpha$ level for statistical significance was 0.05. All analyses were conducted in SPSS version 22.0.

\section{Results}

Within the study period, 50 female patients were admitted to Spring Ward. The mean age was 37.5 years (s.d. 11.17). The sample comprised $20(40 \%)$ White, 26 (52\%) Black and four (8\%) individuals of 'other' ethnicity. The most common primary diagnoses were schizophrenia spectrum disorders $(n=38 ; 78 \%$ of sample) (International Classification of Diseases (ICD-10) category F20-29) and personality disorders (category F60-F69) ( $n=36$ patients, 18\%). Nine patients $(18 \%)$ with a schizophrenia spectrum disorder had a secondary diagnosis of a personality disorder. A diagnosis of a schizophrenia spectrum disorder was associated with non-White ethnicity $\left(\chi^{2}=8.059\right.$, d.f. $\left.=1, P=0.005\right)$, while a diagnosis of personality disorder was associated with White ethnicity $\left(\chi^{2}=4.678\right.$, d.f. $\left.=1, P=0.031\right)$.

A history of physical or sexual abuse or neglect was present in $26(52 \%)$, and $22(44 \%)$ had a history of self-harm. Twenty-three patients (46\%) had a history of alcohol and/or drug misuse, with those of White ethnicity having increased alcohol misuse compared to those of non-White ethnicity (alcohol: $\chi^{2}=6.63$, d.f. $=2, P=0.036$; drugs: $\chi^{2}=7.49$, d.f. $=2$, $P=0.024)$. Eighteen patients $(36 \%)$ had no index offence, $17(34 \%)$ had a violent index offence (actual or grievous bodily harm, manslaughter or murder), eight (16\%) had committed arson and seven (14\%) had a non-violent index offence.

The median length of hospital admission was 503 days (1.39 years). As of November 2014, 46 of the 50 patients were discharged at least once following admission; four were still in hospital following their first admission. Thirteen patients (26\%) were readmitted once or more during the study period. The median follow-up period was 1390 days (3.8 years) for the 46 patients who were discharged. Of this group, five (10.8\%) were discharged to prison; 32 (69.5\%) were subject to a CTO, probation, or section MHA restriction order; and nine (19.5\%) were not subject to conditions or restrictions.

Table 1 shows outcome data on the HCR-20, HoNOS and HoNOS-Secure scales. There were significant improvements on each of these scales at follow-up. Quality of life outcomes on WHOQOL-BREF were 'good' (60-69) for physical (mean 66.2), social (mean 64.3) and environmental domains (mean 67), which compared favourably with international samples of individuals with mental illness. ${ }^{11}$ The mean score for psychological health (mean 58.9) was 'acceptable' (50-59), which was comparable to that of patients with psychotic disorders in international samples. ${ }^{11}$

Of 41 discharges to a hospital or community setting, data on reoffending were available for 40 . In this group, seven patients (17.5\%) reoffended (four with violent offences). Those who reoffended had a significantly shorter hospital stay (mean days 231.7, s.d. 226) compared with those who did not reoffend (mean days 631.7, s.d. $=326 ; t=3.701$, d.f. $=$ $12.66, P=0.005$ ). The length of stay of those who were readmitted did not significantly differ compared with those who were not readmitted $(t=0.215$, d.f. $=27.05, P=0.831)$. Likewise, use of conditions, restrictions or probation was not associated with readmission $\left(\chi^{2}=4.66\right.$, d.f. $\left.=2, P=0.097\right)$, but was associated with reduced reoffending $\left(\chi^{2}=43.14\right.$, d.f. $=6, P<$ 0.001). Alcohol or drug use was not found to be associated with readmission $\left(\chi^{2}=0.02\right.$, d.f. $\left.=1, P=0.887\right)$, but was associated with increased reoffending $\left(\chi^{2}=4.49\right.$, d.f. $\left.=1, P=0.034\right)$.

\begin{tabular}{|lcccccccc|}
\hline Table 1 & HCR-20, HoNOS and HoNOS-Secure scales: mean differences between initial assessment and follow-up assessment \\
& $\begin{array}{c}\text { Initial assessment } \\
\text { mean score }\end{array}$ & $\begin{array}{c}\text { Follow-up assessment } \\
\text { mean score }\end{array}$ & $\begin{array}{c}\text { Mean } \\
\text { difference }\end{array}$ & s.d. & $95 \% \mathrm{Cl}$ & $t$ & $\begin{array}{c}\text { S.f. } \\
\text { (2-tailed) }\end{array}$ \\
\hline HCR clinical items & 6.32 & 3.34 & 2.97 & 3.44 & $1.78-4.15$ & 5.106 & 34 & $<0.001$ \\
\hline HCR risk items & 5.91 & 4.13 & 1.77 & 3.08 & $0.73-2.82$ & 3.462 & 35 & 0.001 \\
\hline HoNOS scale & 15.07 & 7.71 & 7.35 & 16.30 & $2.07-12.64$ & 2.818 & 38 & 0.008 \\
\hline $\begin{array}{l}\text { HoNOS-Secure } \\
\text { scale }\end{array}$ & 15.15 & 4.30 & 10.84 & 20.87 & $4.07-17.61$ & 3.244 & 38 & 0.002 \\
\hline
\end{tabular}




\section{Discussion}

We report clinical characteristics and follow-up outcomes on 50 female MSU patients, a group that has been underrepresented by research to date. Studies of this group have focused primarily on readmission and reoffending only, and there have been few new published data in the past decade. Our study provides an update in this important area of forensic psychiatry, including clinical outcome measures. This is also the first study in this group to include selfreported quality of life data.

A key finding of our study was that women discharged from hospital maintained their improvement for the follow-up period and reported good outcomes. Reduction in clinical and risk items on HCR-20 suggests both clinical improvement for patients and reduction in risk of future violent offending in the community. Overall quality of life measures (WHOQOL-BREF) were grossly comparable to a population of individuals with mental health problems. ${ }^{11}$ Quality of life is an important outcome for patients and their family following discharge. Lower scores on physical and psychological domains were a concern, although this is perhaps to be expected given the complexity of our population.

As with previous studies, we found that a longer hospital stay was associated with a lower rate of reoffending. While forensic services have been criticised for longer periods of stay in hospital, ${ }^{12}$ it has also been argued that this is necessary for patient recovery and public protection. ${ }^{13}$ Our findings provide support for this hypothesis, and are in keeping with results from a previous large-scale study showing that a longer in-patient stay was protective against reoffending following discharge. ${ }^{4}$ The use of restrictions in forensic services is also controversial, but has also been shown to be associated with reduced reoffending following discharge. ${ }^{4}$ This was also the case in our sample. These results suggest that longer stays in hospital and judicious use of restrictions on discharge are necessary to prevent reoffending in this patient group.

In our sample, alcohol and drug use were also associated with increased reoffending. The largest study of reoffending following discharge from MSU settings did not identify this association, demonstrating only an association between alcohol use and arson. ${ }^{4}$ However, this association may also be due to distinct clinical characteristics of our sample and warrants further exploration.

Our sample's demographic characteristics were similar to those of previous studies which have examined female MSU patients. ${ }^{4,5,9,14}$ There were high rates of abuse and neglect, alcohol and substance misuse and self-harm, emphasising that this is a vulnerable group, and supporting the view that there is a need for specialised MSU services for women. ${ }^{2,9}$ There were higher rates of psychotic disorders than in previous samples, and lower rates of personality disorders. ${ }^{4}$ This may be due to a tendency in some settings to diagnose personality disorder, rather than psychosis, on the basis of the presence of self-harm, impulsive behaviour and substance misuse, an issue that has been identified as a concern in clinical guidelines. ${ }^{15}$ Our exclusively urban sample may also contribute to the discrepancy.
There was a high representation of ethnic minorities within our female forensic service. Black women admitted to MSUs have previously been shown to be more likely to have diagnoses of psychotic disorder or drug misuse, and to be less likely to be diagnosed with depression, alcohol misuse and borderline personality disorder than White women. ${ }^{14}$ In the current study, ethnicity was also associated with different clinical profiles in our patient group. Owing to our small sample size, we limited our categorisation of ethnicity to White and non-White. In keeping with previous research, non-White ethnicity was associated with increased rates of schizophrenia spectrum disorders, while White ethnicity was associated with increased rates of personality disorder. ${ }^{14}$ This tentatively supports the hypothesis that there is a deficit in adequately providing appropriate non-forensic services to non-White women with psychotic disorders, leading to escalation to offending and admission to forensic services. Also in keeping with previous studies, ${ }^{14}$ White ethnicity was associated with increased alcohol misuse. However, in contrast to previous findings, White ethnicity in our sample was also associated with increased drug misuse. This suggests a potential shift in clinical characteristics of White female patients being admitted to medium secure settings. Inferences are limited, however, by our small sample size and exclusively urban population.

The study was limited mainly by a relatively small sample size, although this needs to be considered in relation to the relatively small female MSU population, and the lack of relevant data on this group. The study benefited from robust data collection - very few data were missing - and from the use of standardised and objective measures of outcomes.

In conclusion, admission to female MSU is associated with improvement on clinical risk assessment outcomes at follow-up. Longer hospital admissions and the use of restrictions on discharge may be necessary to prevent reoffending in this group. Further research on the role of ethnicity in this group is warranted.

\section{Acknowledgements}

We would like to thank all staff on Spring Ward, River House MSU, for assistance with data collation and acquisition.

\section{About the authors}

John Tully is a Wellcome Clinical Research Training Fellow at the Department of Forensic and Neurodevelopmental Services, Institute of Psychiatry, Psychology and Neuroscience, Kings College London, UK; Alessandra Cappai is a specialist registrar in Forensic Psychiatry at River House MSU, Bethlem Royal Hospital, South London and Maudsley Foundation Trust, UK; John Lally is a clinical research worker at the Department of Psychosis Studies, Institute of Psychiatry, Psychology and Neuroscience, Kings College London and National Psychosis Service, South London and Maudsley NHS Foundation Trust, UK and Department of Psychiatry, Royal College of Surgeons in Ireland, Ireland; and Maria Fotiadou is a consultant forensic psychiatrist at River House MSU, Bethlem Royal Hospital, South London and Maudsley Foundation Trust, UK. 


\section{References}

1 Davies S, Clarke M, Hollin C, Duggan C. Long-term outcomes after discharge from medium secure care: a cause for concern. Br J Psychiatry 2007; 191(1): 70-4.

2 Rutherford M, Duggan S. Forensic mental health services: facts and figures on current provision. Br J Forensic Practice 2008; 10(4): 4-10.

3 Long C, Dolley O, Hollin C. Women in medium secure care: tracking treatment progress for changes in risk profiles and treatment engagement. J Psychiat Ment Health Nurs 2011; 18(5): 425-31.

4 Coid J, Hickey N, Kahtan N, Zhang T, Yang M. Patients discharged from medium secure forensic psychiatry services: reconvictions and risk factors. Br J Psychiatry 2007; 190(3): 223-9.

5 Sahota S, Davies S, Duggan C, Clarke M, Huband N, Owen V. Women admitted to medium secure care: their admission characteristics and outcome as compared with men. Int J Forensic Ment Health 2010; 9(2): 110-7.

6 Douglas KS, Hart SD, Webster CD, Belfrage H. HCR-20v3: Assessing Risk for Violence: User Guide. Mental Health, Law, and Policy Institute, Simon Fraser University, 2013.

7 Dickens G, Sugarman P, Walker L. HoNOS-secure: a reliable outcome measure for users of secure and forensic mental health services. Forensic Psychiatry Psychol 2007; 18(4): 507-14.

8 Gray NS, Hill C, McGleish A, Timmons D, MacCulloch MJ, Snowden RJ. Prediction of violence and self-harm in mentally disordered offenders: a prospective study of the efficacy of HCR-20, PCL-R, and psychiatric symptomatology. J Consult Clin Psychol 2003; 71(3): 443.

9 Long CG, Fulton B, Hollin CR. The development of a 'best practice' service for women in a medium-secure psychiatric setting: treatment components and evaluation. Clin Psychol Psychother 2008; 15(5): 304-19.

10 Harper A. Development of the World Health Organisation WHOQOLBREF quality of life assessment. Psychol Med 1998; 28(3): 551-8.

11 Skevington SM, McCrate FM. Expecting a good quality of life in health: assessing people with diverse diseases and conditions using the WHOQOL-BREF. Health Exp 2012; 15(1): 49-62.

12 Völlm B, Bartlett P, McDonald R. Ethical issues of long-term forensic psychiatric care. Ethics Med Public Health 2016; 2(1): 36-44.

13 Edwards J, Steed P, Murray K. Clinical and forensic outcome 2 years and 5 years after admission to a medium secure unit. J Forensic Psychiatry 2002; 13(1): 68-87.

14 Coid JW, Kahtan N, Gault S, Jarman B. Ethnic differences in admissions to secure forensic psychiatry services. Br J Psychiatry 2000; 177(3): 241-7.

15 National Institute of Health and Care Excellence. Borderline Personality Disorder: Recognition and Management. NICE, 2009 (https://www.nice. org.uk/guidance/cg78).

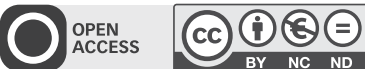

